der deutsch-venezolanischen Gesellschaft am 10./11. Juni 1987 ein Symposium, dessen Beiträge der vorliegende Band zweisprachig präsentiert.

Umrahmt vom authentischen spanischen und französischen Vertragstext nebst deutscher Übersetzung (!) und dem deutsch-venezolanischen Kulturabkommen vom 8. April 1987 vermitteln die Beiträge Eindrücke aus historischer (Guillermo Morón, Hans-J. König, Rolf Walter), rechtsgeschichtlicher (Georg Engelbrecht), wirtschaftlicher (Otto Seiler, Hans J. Hainich, Ulrich Graeser) und kulturell-wissenschaftlicher (Theo Eberhard) Perspektive. Die Vielfalt rundet eine mehr als hundert Titel umfassende Spezialbibliographie zu Venezuela und den venezolanisch-deutschen Beziehungen sinnvoll ab.

Karl-Andreas Hernekamp

De Andrea Sánchez, Newman Valenzuela, Rodríguez Lozano, Sánchez Bringas, Solano Yánez

\title{
La Renovación Política y el Sistema Electoral Mexicano
}

Prólogo Héctor Fix Zamudio, México D.F.: Editorial Porrúa, S.A., 187, 259 S.

Fragen der politischen Emeuerung und des Wahlsystems in Mexico können mit einem so großen Leserkreis rechnen, daß es der angesehene Porrúa-Verlag in Mexiko-Stadt nicht als geschäftliches Wagnis ansah, eine Sammlung mit zehn Beiträgen zu diesem Thema gleich in einer Auflage von 5.000 Exemplaren - in Leinen gebunden - herauszugeben. Nur wer oberflächlich einige der ausführlich behandelten Themen herausgreift, könnte befürchten, die erörterten Fragen sprächen vorwiegend doch nur einen kleinen Kreis von Verfassungsrechtlem, Politikwissenschaftlem und Historikem an; geht es doch auch um Probleme, deren Bedeutung einem Außenstehenden eher rechtstechnisch oder historisch vorkommen mag, wenn beispielsweise von Anhörungen bei wirtschaftlichen und sozialen Entwicklungsplänen, Sitzungsperioden des Kongresses, der Gemeindereform, der Geschichte des mexikanischen Wahlrechts, Fragen des Wählerverzeichnisses oder der Ausgestaltung des repräsentativen Systems die Rede ist. Wer allerdings die behandelten Themen im Zusammenhang würdigt, wird bald jeden Zweifel daran verlieren, daß die Untersuchungen nicht nur für Mexiko von außerordentlicher Bedeutung sind. Ihre unmittelbare Aktualität wird durch das Echo unterstrichen, das die mexikanischen Wahlen im Juli 1988 weltweit gefunden haben. Gerade weil die Berichte darüber, insbesondere auch in den deutschen Medien, von den Protesten der Oppositionsparteien gegen das Wahlverfahren überschattet wurden, verdienen die verfassungsrechtlichen und politischen Grundlagen des Wahlsystems besondere Aufmerksamkeit.

Was die unmittelbaren politischen Auswirkungen angeht, kann es als ein wichtiger Schritt auf einen wirklichen demokratischen Pluralismus angesehen werden, daß - trotz mögli- 
cherweise berechtigter Beanstandungen des Wahlvorganges im einzelnen - die dienstälteste Regierungspartei der westlichen Welt das schlechteste Wahlergebnis seit ihrer Gründung im Jahre 1929 zu verzeichnen hat. Die "offizielle Partei", wie die Revolutionspartei, heute "Partido Revolucionario Institucional" (PRI) genannt, selbst in Lehrbüchern des Verfassungsrechts bezeichnet wird, ist zu einer gewöhnlichen Mehrheitspartei geworden, wie es der künftige Präsident Salinas de Gortari schon vor der Wahl als der politischen Entwicklung angekündigt hatte. Selbst im Senat sind zum ersten Mal seit 59 Jahren Politiker der Opposition vertreten.

In seinem Vorwort macht Héctor Fix Zamudio die Zusammenhänge deutlich, die dem Sammelband unter verschiedenen Gesichtspunkten große Aufmerksamkeit sichem. Der untrennbare Zusammenhang zwischen der sozialen Gerechtigkeit und einer adäquaten politischen Organisation ist ein Leitmotiv des Buches. Es ist der Stolz der Mexikaner, in ihrer Verfassung von 1917 die rein politische und formale Orientierung der Verfassungen des 19. Jahrhunderts überwunden und eingehende Regelungen zum Schutz von Arbeitnehmern und Kleinbauern verankert zu haben, um einen "sozialen Konstitutionalismus" zu verwirklichen.

José Newman Valenzuela - der als Direktor des Nationalen Wahlamtes heftigen Angriffen vor allem von Seiten der Oppositionspartei PAN ausgesetzt ist - befaßt sich eingehend mit dem Funktionieren des Wählerverzeichnisses als einer elementaren Voraussetzung freier Wahlen; er hebt dabei die Bedeutung der Verläßlichkeit und Vertrauenswürdigkeit des Vorgehens hervor. Enrique Sánchez Bringas und Francisco J. de Andrea Sánchez stellen die Entwicklung des Wahlsystems im heutigen Mexiko von der spanischen Verfassung von Cádiz aus dem Jahre 1812 bis zum neuen Delfino Solano Yañez, der sich ausführlich rechtsvergleichenden Untersuchungen widmet.

Ein Ausgangspunkt für eine bessere Partizipation der Bevölkerung ist das seit 1928 entwickelte Verfahren der Anhörung bei der wirtschaftlichen und sozialen Planung, die als "gemischte Wirtschaft" deutlich von den Formen sozialistischer Planwirtschaft abgehoben wird. Besonders in der Zeit von 1982 bis 1987 fanden verschiedenen Anhörungen (consultas populares) statt, die den Weg zu einer demokratischeren Gesellschaft ebnen sollten (Amador Rodríguez Lozano).

Die Verankerung der politischen Parteien in der Verfassung (constitucionalización de los partidos políticos) ist ein besonderer Schwerpunkt des besprochenen Sammelbandes. Bei der umfassenden rechtsvergleichenden Betrachtung wird neben Artikel 21 des Bonner Grundgesetzes auch die badische Verfassung von 1947 nicht vergessen; nur die Rolle des deutschen Bundesverfassungsgerichts bei der Entwicklung des Parteiengesetzes kommt zu kurz. Hervorgehoben wird vor allem die portugiesische Verfassung von 1976, die 22 Artikel ausschließlich den Parteien widmet.

Für die Entwicklung in Mexiko stellten die politischen Reformen von 1977 die entscheidenden Weichen, nachdem die früheren Versuche, Minderheitsparteien zu stärken, nur geringe Erfolge gezeigt hatten. Damals wurde den politischen Parteien in Artikel 41 die Aufgabe übertragen, die Partizipation des Volkes am politischen Leben zu fördern und zur 
"Integration der nationalen Repräsentation" beizutragen. Die Rechte aller Parteien wurden im Interesse der Chancengleichheit - einschließlich des gleichmäßigen Zugangs zu den Medien - eingehend geregelt und dann im Wahlgesetz von 1987 noch näher konkretisiert. Auch die Frage der Parteienfinanzierung wird geregelt. Die Regelungen über die Arbeit der Parteien werden gegenüber mancher Kritik, der sie zu sehr ins einzelne gehen, ausdrücklich befürwortet. Insbesondere will man verhindern, daß Parteien zu trojanischen Pferden werden, die von innen die demokratische Gesetzmäßigkeit gefährden (Pedro Vega).

Die weitere Entwicklung wird zeigen, ob es gelungen ist, entgegen den im Juli 1988 laut gewordenen Protesten und Aufforderungen zum zivilen Ungehorsam, demokratischem Pluralismus und politischer Stabilität näherzukommen. Eine wichtige Rolle wird dabei die Vertrauenswürdigkeit der in dem Buch beschreibenen Vorkehrungen zur Überprüfung der Ordnungsmäßigkeit der Wahlen spielen (Enrique Sánchez Bringas).

Hans-Rudolf Horn

Assoziazione di Studi Sociali Latino-Americani/Casa de Bello (Hrsg.)

Andrés Bello y el Derecho Latinoamericano

Caracas 1987, $530 \mathrm{~S}$.

Das eindrucksvolle und immer noch erstaunlich aktuelle Lebenswerk des großen Humanisten und Rechtsdenkers Andrés Bello, der 1781 in Caracas/Venezuela geboren und 1865 in Santiago de Chile gestorben ist, war Gegenstand eines internationalen Kongresses, der vom 10. bis 12. Dezember 1981 in Rom stattfand. Veranstalter waren die italienische Assoziazione di Studi Sociali Latino-Americani (ASSLA), das eigens zum 200. Geburtstag ins Leben gerufene venezolanische Nationalkomitee unter Leitung des früheren Staatspräsidenten Caldera und die Stiftung Casa de Bello.

30 Fachbeiträge von Autoren aus Italien, Venezuela, Chile, Kolumbien, Uruguay, Spanien, Frankreich und der Bundesrepublik Deutschland, der Schweiz und Osterreich sowie der Text von sechs Ansprachen der Veranstalter, die ebenfalls auf das Werk und die Person Bellos eingingen, sind auf Spanisch oder Italienisch in dem vorliegenden Band "Andrés Bello y el Derecho Latinoamericano" zusammengefaßt.

Leitmotiv der Beiträge, die sich durch ein hohes wissenschaftliches Niveau auszeichnen, ist die Entwicklung eines eigenständigen Rechtssystems aus der politischen Wirklichkeit der neu entstandenen Staaten Lateinamerikas. Der Abschnitt "Andrés Bello und das Völkerrecht in Lateinamerika" steht sicher nicht zufällig an erster Stelle (S. 23-169): hier liegen sicher die Hauptverdienste des vielseitigen Juristen. Vom äußeren Umfang her ist freilich selbst der Abschnitt "Universität und römisches Recht im Rahmen der Juristenausbildung" größer, wenn man den Beitrag Vergilio Ilaris über den Zusammenhang zwischen dem römi- 